\title{
Demonstration of improved effectiveness factor of catalysts based on hollow single crystal zeolites
}

\author{
Céline Pagis, Frédéric Meunier, Yves Schuurman, Alain Tuel*, Mathias Dodin, Raquel Martinez-Franco, \\ David Farrusseng
}

\begin{abstract}
Pt/NaY zeolites with bulk and hollow morphologies have been used as model catalysts in the hydrogenation of cyclohexene as model reaction. In contrast to most hierarchical zeolites, the presence of a single internal cavity modifies only the mean diffusion length, without changing the external surface area and the crystal size. Differences in catalytic activities have been attributed to a shorter diffusion length in hollow crystals. The volume fraction of zeolite used in bulk crystals (ca. 0.63-0.70) matches well with the shell thickness in hollow crystals, leading to an efficiency factor value for the latter close to 1 .
\end{abstract}

Zeolites are crystalline microporous aluminosilicates whose structure contains channels, voids and cavities of molecular dimensions, typically between 0.5 and $0.8 \mathrm{~nm}$ in size. Historically, research in zeolite synthesis was largely motivated by their unique and outstanding properties as catalysts, ionexchangers and adsorbents. ${ }^{[1-4]}$ Size and shape of micropores control molecules accessibility to active sites, allowing zeolites to enable unique catalytic properties such as "shape selectivity" ${ }^{[5-8]}$ However, sub-nanometric micropores also influence internal mass transfer and reactions involving molecules with a size similar to the pore opening are very often limited by mass transport. ${ }^{[9-11]}$ Because of this, active sites located far from the surface of large crystals do not significantly participate to the reaction and in effect only a small fraction of zeolite crystals is used, leading to a low "effectiveness factor". ${ }^{[12-14]}$ Molecular transport throughout the zeolite structure and, consequently, the effectiveness factor, can be improved by minimizing the mean diffusion length in individual crystals. This can be achieved by using crystals with a nanometric size, for example zeolite nanosheets or by facilitating the access to internal sites via the formation of mesopores throughout the whole crystal. ${ }^{[15-20]}$ Zeolites combining arrays of connected meso and micropores, also named "hierarchical zeolites" have shown better catalytic performance than bulk crystals in reactions involving large molecules. ${ }^{[21-24]}$ However, the origin of the higher activity of hierarchical zeolites is often under debate ${ }^{\left[{ }^{9]}\right.}$ When comparing a set of zeolites for acid catalyzed reactions, the general assumption made is that only hierarchical porous features change while the other structural features remain the same. However, the presence of mesopores also increases the

\footnotetext{
* C. Pagis, Dr. F. Meunier, Dr. Y. Schuurman, Dr. A. Tuel, Dr. D. Farrusseng

Université de Lyon, Université Claude Bernard Lyon 1, CNRS,

IRCELYON - UMR 5256, 2 Avenue Albert Einstein, 69626 Villeurbanne

Cedex, France

E-mail: alain.tuel@ircelyon.univ-lyon1.fr

C. Pagis, Dr. M. Dodin, Dr R. Martinez-Franco

IFP Energies Nouvelles, Etablissement de Lyon, BP3, 69360 Solaize,

France
}

Supporting information for this article is given via a link at the end of the document.((Please delete this text if not appropriate)) external surface area and as consequence one can hardly attribute the origin of the higher activity either to shorter diffusion length or to larger external surface area / higher pore connectivity. ${ }^{[25]}$ Fortunately, the morphology of "hollow zeolites", also called "nano-boxes" overcomes this trade-off "shorter diffusion length-higher surface area" as the diffusion length varies like the thickness of the walls, while the external surface remains unchanged (Scheme 1). These model zeolite structures that do not possess micropore-mesopore connectivity will allow unravelling the origin of observed higher "effectiveness factor" with respect to the pore structural features. Another direct consequence of the hollow morphology is that the "effectiveness factor" can be enhanced as the thickness of the zeolite wall is reduced, which is particularly relevant for expensive catalysts consisted of supported metals.

The "hollowing" zeolite process has been widely reported for zeolites with MFI framework type, leading to nanoboxes with regular zeolitic walls whose thickness can be controlled down to a few nanometers. ${ }^{[26-30]}$ More recently, we succeeded in preparing hollow Beta and $\mathrm{Y}$ zeolite crystals, with *BEA and FAU framework types, respectively. ${ }^{[31-34]}$

The present work demonstrates that supporting $\mathrm{Pt}$ nanoparticles in hollow $Y$ zeolite crystals enables to reach effectiveness factors close to $100 \%$ in a model hydrogenation reaction, therefore optimizing the metal utilization in the catalyst.

Hollow $\mathrm{Y}$ zeolite crystals were obtained from a standard $\mathrm{NaY}$ zeolite $(\mathrm{Si} / \mathrm{Al}=2.5)$ following a multi-step post-synthesis process involving dealumination by $\mathrm{SiCl}_{4}$ (step 1), leaching with hydrochloric acid (step 2) and finally desilication in the presence of protective Al species (step 3), as reported in one of our previous publications. ${ }^{[34]}$ In the following, the term "bulk" refers to a solid recovered after the second step, just before formation of the internal cavity by selective desilication. Platinum nanoparticles (NPs) were incorporated in bulk NaY crystals $(\mathrm{Si} / \mathrm{Al}=38)$ and their corresponding hollow counterparts (see ESI). The corresponding catalysts denoted Pt@bulk $Y$ and Pt@hollow $\mathrm{Y}$, respectively, possess a Pt loading of 1 wt.\%, similar to that of a commercial reference $\mathrm{Pt} / \mathrm{Al}_{2} \mathrm{O}_{3}$ catalyst (Table 1 and Table S1).

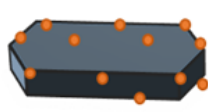

$\mathrm{Pt}_{\mathrm{Al}} \mathrm{O}_{3}$

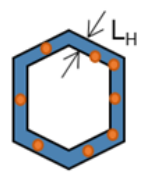

Pt@hollow Y

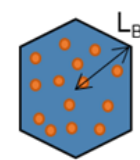

Pt@bulk Y

$$
L_{H}=0.12 \pm 0.05 \mu \mathrm{m}
$$$$
L_{B}=0.6 \pm 0.12 \mu \mathrm{m}
$$

Scheme 1. Schematic representation of the various materials and their corresponding characteristic length. 
Table 1. Composition and textural properties of Pt-loaded bulk and hollow NaY samples. Metal particle size and dispersion are based on the corresponding $\mathrm{d}_{\mathrm{Sw}}{ }^{[\mathrm{a}]}$

\begin{tabular}{cccc}
\hline Catalyst & $\begin{array}{c}\text { Pt loading } \\
{[\mathrm{wt} \%]}\end{array}$ & $\begin{array}{c}\text { Mean particle size } \\
{[\mathrm{nm}]^{[\mathrm{aa}]}}\end{array}$ & $\begin{array}{c}\text { Pt dispersion } \\
{[\%]^{[\mathrm{a}]}}\end{array}$ \\
\hline $\mathrm{Pt} / \mathrm{Al}_{2} \mathrm{O}_{3}$ & 1.0 & $5.4 \pm 1$ & $25 \pm 4$ \\
$\mathrm{Pt} @$ bulk Y & 0.99 & $3.9 \pm 1.2$ & $34 \pm 7$ \\
$\mathrm{Pt} @$ hollow Y & 0.99 & $2.2 \pm 0.5$ & $53 \pm 8$ \\
\hline
\end{tabular}

[a] Surface-weighted mean diameter (precision is based on the standard deviation of the distribution - 400 particles counted)

TEM pictures show that both Pt-loaded zeolite crystals are approx. $1.2 \pm 0.24 \mu \mathrm{m}$ in size and that Pt@hollow differs from Pt@bulk mainly by the presence of an internal cavity of $0.9 \pm 0.1$ $\mu \mathrm{m}$ (Figure 1).

Metal nanoparticles are visible exclusively inside the porosity of the zeolite, probably at structural defects created during the impregnation/reduction steps, and are homogeneously distributed according to TEM observations (Figure 1, Figure S1, Figure S2). Moreover, XPS analyses give surface $\mathrm{Pt} / \mathrm{Si}$ ratios of 0.004 for Pt@hollow $\mathrm{Y}$ and Pt@bulk $\mathrm{Y}$ and 0.06 for $\mathrm{Pt} / \mathrm{SiO}_{2}$ (Figure S3 and Table S2). The factor 15 between the two values confirms that most of $\mathrm{Pt}$ nanoparticles are absent from the zeolite surface and dispersed inside the porosity.

Mean particle sizes calculated from a population of 400 particles are also similar for all catalysts, with surface-weighted mean diameters $d_{s w}$ of 2.2, 3.9, and 5.4 nm for Pt@hollow $Y$, Pt@bulk Y, and $\mathrm{Pt} / \mathrm{Al}_{2} \mathrm{O}_{3}$, respectively (Table 1). This corresponds to metal dispersions ranging from $25 \%$ for $\mathrm{Pt} / \mathrm{Al}_{2} \mathrm{O}_{3}$ to 53 \% for Pt@hollow Y zeolite. Nitrogen adsorption isotherms at $77 \mathrm{~K}$ are similar to those previously reported for Pt-free bulk and hollow Y zeolites. ${ }^{[34]}$ For Pt@hollow Y, the presence of a H2 hysteresis loop with a closure point at $\mathrm{p} / \mathrm{p}_{0}=0.47$ on the desorption branch indicates that the internal cavity is accessible only via entrances smaller than $4 \mathrm{~nm}$, pointing towards an external defect-free purely microporous zeolite shell (Figure S4). ${ }^{[35,36]}$ The absence of hysteresis for the bulk sample clearly demonstrates that the cavity is created during the third step of the preparation method. The three different catalysts used in the present study are summarized in Scheme 1 along with their respective characteristic lengths $L$. Basically, $L$ can be defined as the minimum distance that a molecule has to travel to reach all catalytic sites in the solid.

Assimilating bulk crystals to spheres with an average diameter $D=1.2 \mu \mathrm{m}, L$ corresponds to the radius $L_{B}=D / 2=0.6$ $\mu \mathrm{m}$. In the case of hollow crystals, $L_{H}$ is reduced to the shell thickness, which is $0.12 \mu \mathrm{m}$ as estimated from TEM pictures. Of course, this definition of the characteristic length implies that $\mathrm{Pt}$ is homogeneously distributed throughout the crystals, which appears to be the case from TEM pictures (Figure S1 and Figure S2).

The $\mathrm{Pt} / \mathrm{Al}_{2} \mathrm{O}_{3}$ catalysts consist of an agglomerate of nanosized particles that form a mesopore network. By evaluating the Weisz-Prater criterion, internal mass transfer limitations could be excluded (see ESI).
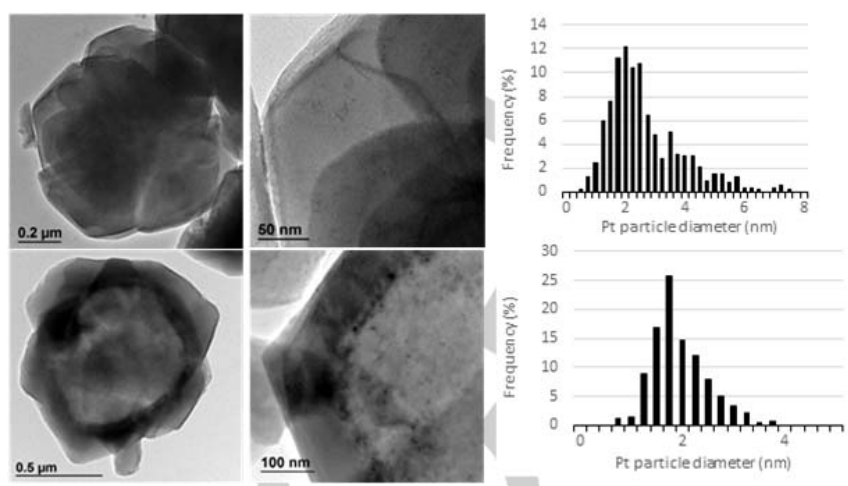

Figure 1. TEM images and corresponding particle size distributions of Pt@bulk Y (top) and Pt@hollow Y (bottom).

The influence of the hollow structure on reaction kinetics was investigated using the model reaction of cyclohexene hydrogenation and discussed in terms of Thiele modulus and effectiveness factor, which are standard indicators for the presence or the absence of diffusion limitations in the zeolite.

Kinetic data were obtained at steady-state conditions following an induction period associated with a significant drop of activity, attributed to site poisoning. ${ }^{[37,38]}$ Conversions were kept lower than $20 \%$, ensuring differential conditions were met (see ESI).

Apparent TOF (formally site-time-yields STY) values for cyclohexene hydrogenation are given in Figure 2. Interestingly, the TOF's are essentially identical for the $\mathrm{Pt} / \mathrm{Al}_{2} \mathrm{O}_{3}$ and the Pt@hollow $Y$ below 80드. while the TOF for the Pt@hollow Y appears to be lower at higher temperature. This behavior could suggest that the apparent TOF becomes limited by mass transport limitation at the higher temperatures. This observation is supported by noting that the TOF measured in the case of the Pt@bulk Y sample is significantly lower at all temperatures, and even more so at higher temperatures, stressing the occurrence of mass transport limitations for this sample.

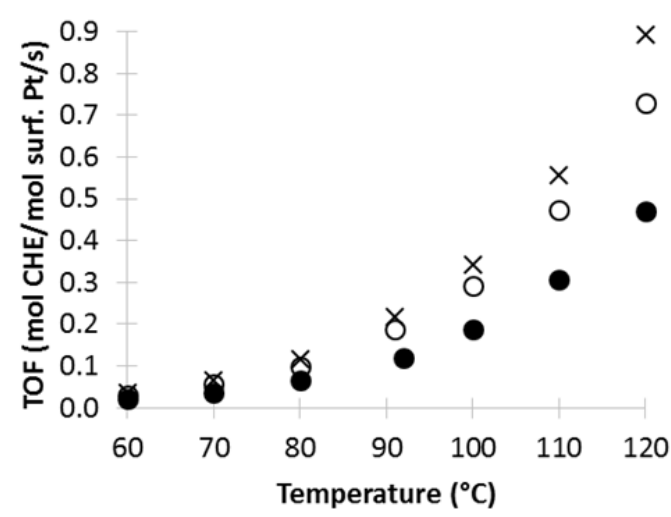

Figure 2. Cyclohexene apparent turn over frequencies (TOF) measured over (x) $\mathrm{Pt} / \mathrm{Al}_{2} \mathrm{O}_{3},(\bullet) \mathrm{Pt} @$ bulk $\mathrm{Y}$ and $(\mathrm{O}) \mathrm{Pt} @$ hollow $\mathrm{Y}$. Feed $=3.3 \%$ in $\mathrm{H}_{2}$, total flow $=20 \mathrm{~mL} \mathrm{~min}^{-1}$ 
The supply of reactants to the active sites can slow down the catalyst performance when the catalytic active sites are located in small pores and a fast chemical reaction takes place.

The ratio of the reaction rate and the transport rate of reactants by pore diffusion is given by the well-known Thiele modulus. ${ }^{[39]}$ For a $\mathrm{n}^{\text {th }}$-order irreversible reaction the Thiele modulus is given by Equation (1): ${ }^{[40]}$

$\phi=L \sqrt{\frac{n+1}{2} \frac{r\left(C_{A s}\right) \rho_{S}}{D_{e f f} C_{A s}}}$

where $L$ is the mean diffusion length related to the particle size, $r\left(C_{A s}\right)$ the reaction rate per mass of catalyst at the particle surface, $\rho_{s}$ the particle density, $D_{\text {eff }}$ the effective pore diffusion coefficient and $C_{A s}$ the reactant concentration at the particle surface. For a given catalyst geometry (sphere, cylinder, slab), the Thiele modulus is related to the catalyst effectiveness factor $\eta$, which is the ratio of the reaction rate with pore diffusion to the reaction rate at the particle surface. If the Thiele modulus is much smaller than 1 , the effectiveness factor is close to one. Otherwise, low effectiveness factors are due to the slow transport of molecules in the pores with respect to the reaction rate. The Thiele modulus is based on the surface reaction rate, which is the rate in absence of diffusion limitations. To evaluate the Weisz-Prater criterion prior information on the value of the diffusion coefficient is necessary. While this can be estimated for molecular and Knudsen diffusion in mesopores, no such data exists for diffusion in micropores. The scarce experimental data sets of hydrocarbon diffusion in zeolites show values that range over several orders of magnitude, depending on the crystal size and morphology as well as on the specific technique that was used to measure the diffusivity. ${ }^{[41]}$ In the current case the reaction rates have been measured over different catalyst particle sizes under identical conditions and we can use the ratio of the observed rates to extract the Thiele modulus and the effectiveness factor thereby eliminating the diffusion coefficient from the equation. ${ }^{[42,43]}$ In fact one can write for the ratio of the two experimental reaction rates [Eq. (2)]:

$$
\frac{r_{e 1}}{r_{e 2}}=\frac{\eta_{1}}{\eta_{2}}
$$

where $r_{e i}$ are the experimentally observed reaction rates. According to the particle shape and type of reaction one substitutes the corresponding expression between the effectiveness factor and the Thiele modulus. For LangmuirHinshelwood kinetics no such analytical expression exists, therefore as a first approximation, we have taken the often used expression for generalized geometries [Eq. (3)] ${ }^{[9,21,44]}$

$$
\eta=\frac{\tanh (\phi)}{\phi}
$$

The effectiveness factor is presented in Figure 3 by the black dotted curve.

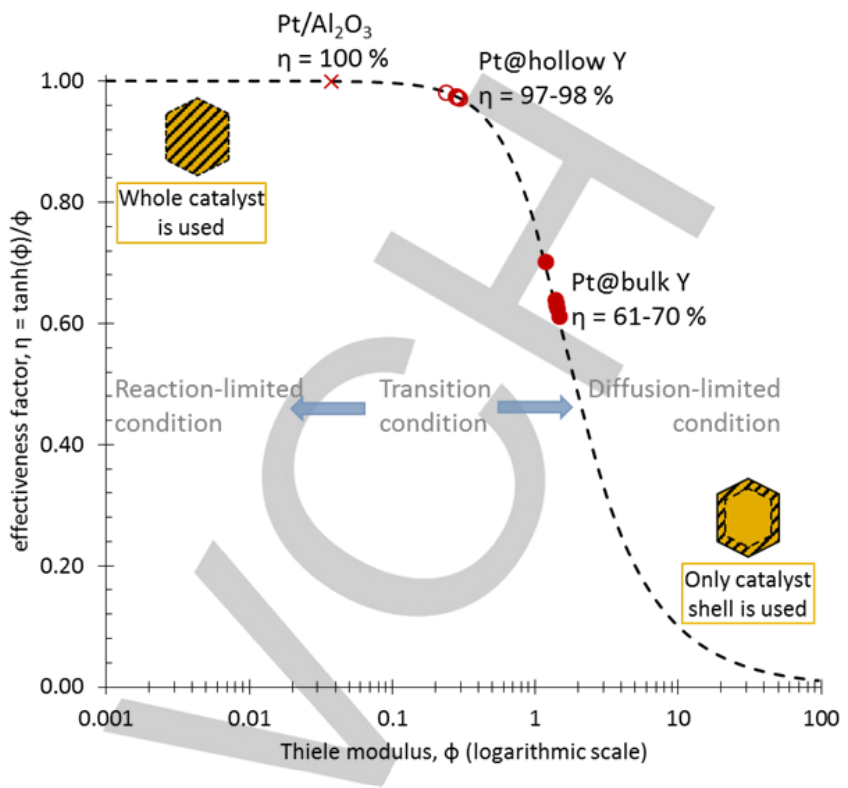

Figure 3. Effectiveness factor as a function of Thiele modulus for the considered catalysts at different reaction temperatures.

Since the rate data have been measured under identical conditions over the same zeolite framework (only the crystal shape is different), it follows from equation (1) that $\phi_{1} / \phi_{2}=L_{1} / L_{2}$. When we substitute this ratio and equation (3) into equation (2) the result is implicit equation with the Thiele modulus as the only unknown parameter.

We have applied this approach to data obtained for the hydrogenation of cyclohexene over the hollow and bulk zeolites described above. The ratio of $L_{1} / L_{2}\left(L_{B} / L_{H}\right.$ in the present case) was set at 5.0, as determined from geometrical considerations and TEM measurements (Scheme 1). Solving equation (2) with the rates based on the TOF values to correct for the difference in the platinum dispersion, the Thiele moduli obtained at $120^{\circ} \mathrm{C}$ for the hollow and bulk samples are 0.28 and 1.41 , respectively (Table 2). The corresponding effectiveness factors are 0.97 and 0.63 , as shown in Table 2 and Fig. 3. For the reference catalyst, the values are calculated by equality between the Weisz-Prater criterion and $\eta \phi^{2}$, considering $L=5 \mu \mathrm{m}$. Considering bulk crystals as spheres with a diameter of $1.2 \mu \mathrm{m}$, an effectiveness factor of 0.63 (i.e. only $63 \%$ of the volume is really used in the reaction) means that the reaction really occurs within a surface layer of approx. $0.15 \mu \mathrm{m}$ thickness. Hollow crystals with a shell thickness smaller than $0.15 \mu \mathrm{m}$ are thus expected to possess an effectiveness factor close to 1 , which is consistent with our experimental observation ( $\eta>0.97$ for $0.12 \mu \mathrm{m}$ ). Calculations for all reaction temperatures give the different values of the Thiele modulus and efficiency factors reported in Figure 3. From the values of the Thiele modulus, a value of the micropore diffusion coefficient of cyclohexene of $4 \times 10^{-12} \mathrm{~m}^{2} / \mathrm{s}$ at $120^{\circ} \mathrm{C}$ was calculated and an activation energy of diffusion of $50 \mathrm{~kJ} / \mathrm{mol}$. As mentioned above no values for the micropore diffusion coefficient for cyclohexene in $Y$ zeolite are reported in the literature. However, the value agrees with $3.6 \times 10^{-12} \mathrm{~m}^{2} / \mathrm{s}$ for 
benzene diffusion in $\mathrm{NaX}$, measured by the zero-length column (ZLC) method. ${ }^{[45]}$

Table 2. Estimated Thiele Modulus and Effectiveness factor from kinetics data $\left(L_{B} / L_{H}=5.0\right)$.

\begin{tabular}{ccccc}
\hline Catalyst & $\begin{array}{c}\text { observed rate } \\
{\left[\mathrm{mmol} / \mathrm{kg}_{\mathrm{cat}} / \mathrm{s}\right]}\end{array}$ & $\begin{array}{c}\text { observed TOF } \\
{\left[\mathrm{mol} / \mathrm{mol}_{\text {Pt.sur }} / \mathrm{s}\right]^{[\mathrm{a}]}}\end{array}$ & $\phi$ & $\eta[\%]$ \\
\hline $\mathrm{Pt} / \mathrm{Al}_{2} \mathrm{O}_{3}$ & 11.5 & 0.89 & 0.037 & 100 \\
$\mathrm{Pt} @$ bulk Y & 8.1 & 0.47 & 1.41 & 63 \\
$\mathrm{Pt} @$ hollow $\mathrm{Y}$ & 19.7 & 0.73 & 0.28 & 97 \\
\hline
\end{tabular}

[a] Values measured at $120^{\circ} \mathrm{C}$

In contrast to standard desilication or crystal size reduction, the formation of hollow structures enables to improve molecular transport in zeolites by reducing the mean diffusion length $L$ without changing other parameters such as the external surface area and the crystal shape. By comparing Pt-supported bulk and hollow $\mathrm{NaY}$ zeolites in the hydrogenation of cyclohexene, it was possible to calculate effectiveness factors and Thiele moduli for both geometries just by comparing their respective $L$ values estimated from TEM images. The effectiveness factor, which represents the fraction of the crystal really used in the reaction, increased from $63 \%$ to more than $97 \%$ in the presence of an internal cavity. In this paper we also provide a methodological approach to estimate the Thiele Modulus for reactions involving zeolites with the same structure and composition without measuring diffusion coefficients of reactants and products. Since the present approach does not depend on the geometry of the micropores, it can be applied to any zeolite structure provided that the reaction is limited by diffusion. Applications using model hollow beta and faujasite zeolites to acid and bifunctional catalytic reactions is under investigation in our laboratory.

\section{Experimental section}

\section{Samples Synthesis and Basic Characterization}

Hollow $\mathrm{NaY}$ was prepared using a three-step process reported in one of our previous publications. ${ }^{[34]}$ Basically, standard $\mathrm{NaY}$ crystals were first dealuminated by silicon tetrachloride (step 1), then leached with hydrochloric acid (step 2) and finally desilicated in the presence of protective Al species (step 3). The term "bulk" refers to a solid recovered after the second step, just before formation of the internal cavity by selective desilication.

Platinum was introduced in both hollow and bulk crystals by incipient wetness impregnation (IWI). Typically, $0.243 \mathrm{~mL}$ of an aqueous solution of $\mathrm{Pt}\left(\mathrm{NH}_{4}\right)_{3}\left(\mathrm{NO}_{3}\right)_{2}$ (99\%, stream chemicals) with a concentration of $0.211 \mathrm{~mol} / \mathrm{L}$ was added to $1 \mathrm{~g}$ of $\mathrm{Y}$ zeolite, which had been calcined at $500{ }^{\circ} \mathrm{C}$ overnight. The mixture was stirred at room temperature for 10 minutes, left static overnight and finally dried at $120{ }^{\circ} \mathrm{C}$ for 3 hours. Then the solid was reduced at $300{ }^{\circ} \mathrm{C}$ under $\mathrm{H}_{2}\left(50 \mathrm{~cm}^{3} / \mathrm{min}\right)$ for $2 \mathrm{~h}$ with a heating rate of $5{ }^{\circ} \mathrm{C} / \mathrm{min}$ to yield samples further denoted Pt@bulk $Y$ and Pt@hollow Y, for bulk and hollow zeolite crystals, respectively.

$\mathrm{Pt} / \mathrm{Al}_{2} \mathrm{O}_{3}$ from Sigma-Aldrich (CAS 232114, 1 wt.\% Pt, Pt dispersion: 0.25 , BET surface area: $206 \mathrm{~m}^{2} / \mathrm{g}$ ) was crushed to obtain a powder and used as a reference catalyst. In contrast to zeolite-based catalyst, all Pt nanoparticles are located on the external surface of the solid and directly accessible to molecules. Likewise, $\mathrm{Pt} / \mathrm{SiO}_{2}$ from Johnson Matthey (powder, 1,8 wt.\% Pt) was used as reference catalyst for XPS analyses.

Solids were characterized by nitrogen adsorption at $77 \mathrm{~K}$ on a Belsorp-mini (BEL-Japan) sorption apparatus. Circa $50 \mathrm{mg}$ of sample was outgassed under vacuum in a cell at $300{ }^{\circ} \mathrm{C}$ overnight prior to adsorption.

Transmission electron microscopy (TEM) images were obtained on a JEOL $2010 \mathrm{LaB}_{6}$ microscope operating at $200 \mathrm{kV}$. A dispersion of the sample crushed in ethanol was deposited on standard holey carbon-covered copper TEM grids. The platinum content in the zeolites was obtained by ICP-OES on an Activa spectrometer (Horiba, Jobin Yvon) equipped with a CCD detector for the determination of metal loading.

XPS analyses were carried out using a commercial Kratos AXIS Ultra DLD spectrometer with a monochromatic AI Ka X-ray source at $1486.6 \mathrm{eV}$. Each high-resolution spectrum was decomposed into a combination of Voigt functions (a Voigt function is a 70/30 composition of Gaussian and Lorentzian functions), each with an overall full-width at half maximum (FWHM) of approximately $1.8 \mathrm{eV}$.

\section{Catalytic Hydrogenation of Cyclohexene}

Hydrogenation tests were carried out using a tubular quartz plug flow reactor placed in a tubular furnace at atmospheric pressure. The reactor effluent was analyzed using a gas cell fitted in a Bruker Tensor 27 FT-IR spectrophotometer. All reaction parameters and analysis procedures are detailed in the ESI.

Keywords: hollow zeolite - heterogeneous catalysis - kinetics • transport $\cdot$ effectiveness factor

[1] S. Kesraoui-Ouki, C. R. Cheeseman, R. Perry, J. Chem. Technol. Biotechnol. 1994, 59, 121-126.

[2] C. Martínez, A. Corma, Coord Chem. Rev. 2011, 255, 1558-1580.

[3] K. Tanabe, Appl. Catal. 1999, 181, 399-434.

[4] P. B. Venuto, Microporous Mater. 1994, 2, 297-411.

[5] W. Vermeiren, J.-P. Gilson, Top. Catal. 2009, 52, 1131-1161.

[6] J. Xiao, J. Wei, Chem. Eng. Sci. 1992, 47, 1123-1141.

[7] B. Smit, T. L. M. Maesen, Nature 2008, 451, 671-678.

[8] E. Derouane, J. Catal. 1980, 65, 486-489.

[9] M. Hartmann, A. G. Machoke, W. Schwieger, Chem. Soc. Rev. 2016, 45 3313-3330.

[10] J. Kärger, S. Vasenkov, Microporous Mesoporous Mater. 2005, 85, 195-206.

[11] K. Hahn, J. Kärger, V. Kukla, Phys. Rev. Lett. 1996, 76, 2762-2765.

[12] G. W. Roberts, C. N. Satterfield, Ind. Eng. Chem. Fundam. 1965, 4, 288-293.

[13] H. Konno, T. Okamura, T. Kawahara, Y. Nakasaka, T. Tago, T. Masuda, Chem. Eng. J. 2012, 207-208, 490-496.

[14] J. O. Mingle, J. M. Smith, AIChE J. 1961, 7, 243-249.

[15] L. Tosheva, V. P. Valtchev, Chem. Mater. 2005, 17, 2494-2513.

[16] S. Mintova, M. Jaber, V. Valtchev, Chem. Soc. Rev. 2015, 44, 72077233.

[17] M. Choi, K. Na, J. Kim, Y. Sakamoto, O. Terasaki, R. Ryoo, Nature 2009, 461, 246-249.

[18] C. J. H. Jacobsen, C. Madsen, J. Houzvicka, I. Schmidt, A. Carlsson, J. Am. Chem. Soc. 2000, 122, 7116-7117. 
[19] K. Egeblad, C. H. Christensen, M. Kustova, C. H. Christensen, Chem. Mater. 2008, 20, 946-960.

[20] F. C. Meunier, D. Verboekend, J.-P. Gilson, J. C. Groen, J. Pérez Ramírez, Microporous Mesoporous Mater. 2012, 148, 115-121.

[21] J. Pérez-Ramírez, C. H. Christensen, K. Egeblad, C. H. Christensen, J. C. Groen, Chem. Soc. Rev. 2008, 37, 2530.

[22] C. M. A. Parlett, K. Wilson, A. F. Lee, Chem Soc Rev 2013, 42, 38763893.

[23] M. Hartmann, Angew. Chem. Int. Ed. 2004, 43, 5880-5882.

[24] M. S. Holm, E. Taarning, K. Egeblad, C. H. Christensen, Catal. Today 2011, 168, 3-16.

[25] X. Zhang, D. Liu, D. Xu, S. Asahina, K. A. Cychosz, K. V. Agrawal, Y. Al Wahedi, A. Bhan, S. Al Hashimi, O. Terasaki, Science 2012, 336, 16841687.

[26] J. C. Groen, T. Bach, U. Ziese, A. M. Paulaime-van Donk, K. P. de Jong J. A. Moulijn, J. Pérez-Ramírez, J. Am. Chem. Soc. 2005, 127, 1079210793.

[27] Y. Wang, M. Lin, A. Tuel, Microporous Mesoporous Mater. 2007, 102, $80-85$

[28] Y. Wang, A. Tuel, Microporous Mesoporous Mater. 2008, 113, 286-295.

[29] C. Mei, Z. Liu, P. Wen, Z. Xie, W. Hua, Z. Gao, J. Mater. Chem. 2008 $18,3496$.

[30] C. Pagis, A. R. Morgado Prates, D. Farrusseng, N. Bats, A. Tuel, Chem. Mater. 2016, 28, 5205-5223.

[31] A. R. Morgado Prates, C. Pagis, F. C. Meunier, L. Burel, T. Epicier, L. Roiban, S. Koneti, N. Bats, D. Farrusseng, A. Tuel, Cryst. Growth Des. 2018, 18, 592-596.

[32] D. Yuan, C. Kang, W. Wang, H. Li, X. Zhu, Y. Wang, X. Gao, B. Wang, H. Zhao, C. Liu, Catal. Sci. Technol. 2016, 6, 8364-8374.

[33] K. lyoki, K. Itabashi, T. Okubo, Chem. - Asian J. 2013, 8, 1419-1427.

[34] C. Pagis, A. R. Morgado Prates, N. Bats, A. Tuel, D. Farrusseng, CrystEngComm 2018, 20, 1564-1572.

[35] J. Groen, Appl. Catal. 2004, 268, 121-125.

[36] M. Thommes, K. Kaneko, A. V. Neimark, J. P. Olivier, F. RodriguezReinoso, J. Rouquerol, K. S. W. Sing, Pure Appl. Chem. 2015, 87, DOI 10.1515/pac-2014-1117.

[37] M. Boudart, C. M. McConica, J. Catal. 1989, 117, 33-41.

[38] D. E. Gardin, X. Su, P. S. Cremer, G. A. Somorjai, J. Catal. 1996, 158 193-198.

[39] E. W. Thiele, Ind. Eng. Chem. 1939, 31, 916-920.

[40] G. F. Froment, J. De Wilde, K. B. Bischoff, Chemical Reactor Analysis and Design, Wiley, Hoboken, N.J, 2011.

[41] J. Kärger, D. M. Ruthven, D. N. Theodorou, Diffusion in Nanoporous Materials, Wiley-VCH, Weinheim, Germany, 2012.

[42] H. S. Fogler, Elements of Chemical Reaction Engineering, Prentice Hall PTR, Upper Saddle River, N.J, 1999.

[43] J. M. Smith, Chemical Engineering Kinetics, McGraw-Hill, New York 1981.

[44] H. Konno, T. Okamura, T. Kawahara, Y. Nakasaka, T. Tago, T. Masuda, Chem. Eng. J. 2012, 207-208, 490-496.

[45] M. Eic, M. Goddard, D. M. Ruthven, Zeolites 1988, 8, 327-331. 
Entry for the Table of Contents (Please choose one layout)

Layout 1:

\section{COMMUNICATION}

No core needed! Why

keep unused parts of catalysts? Removing the poorly accessible core of $\mathrm{NaY}$ crystals supporting Pt enabled the increase the effectiveness factor of cyclohexene hydrogenation from 0.63 to 0.97 , thus limiting the need of rare and expensive noble metals.

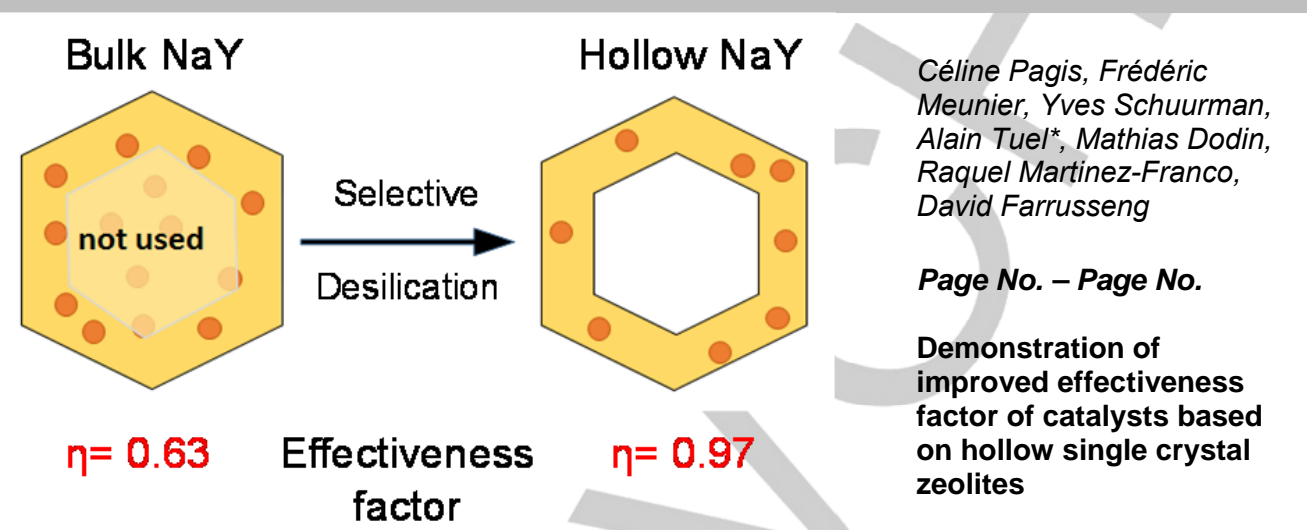

